\title{
ARE AAGBI ANTISEPSIS GUIDELINES FOR CENTRAL NEURAXIAL BLOCKADE (CNB) EFFECTIVE FOR CAUDAL EPIDURAL INJECTIONS IN ADULTS
}

\author{
A. Hania ${ }^{1}$, T. O'Connor ${ }^{1}$.
}

${ }^{1}$ Sligo University Hospital, Pain, Sligo, Ireland.

\section{Introduction}

The AAGBI Guidelines recommends $0.5 \%$ solution of chlorhexidine in alcohol for skin antisepsis before central neuraxial block (CNB) (1). CNB also includes caudal epidural injections. The efficacy of $0.5 \%$ chlorhexidine has not been studied extensively in the sacrococcygeal area. It has been reported that the incidence of epidural catheter tip colonisation is increased in the caudal site when compared to the lumbar site(2). Epidural abscess is a well known complication of epidural catheter insertion that can lead to paralysis (3).

Our aim was to assess the efficacy of $0.5 \%$ chlorhexidine and $70 \%$ alcohol in the sacrococygeal membrane prior to caudal epidural injection.

\section{Materials and methods}

Skin surface samples were taken from 11 patients that attended the Pain Clinic in Sligo Hospital from the sacrococcygeal area following administration of chlorhexidine $0.5 \%$ and $70 \%$ denatured alcohol (Hydrex) prior to caudal epidural injection. The skin was allowed to dry and skin cuture samples were taken by sterile cotton swabs and were inoculated in blood agar media and incubated for $48 \mathrm{hrs}$ in microbiology laboratory.

\section{All patients gave informed written} consent prior to the procedure when consenting for their caudal epidural injection.

\section{Results}

Data from all 11 patients showed no growth after incubation for $48 \mathrm{hrs}$. 2 of the patients had significant hair in the region of the sacrococcygeal area.

\begin{tabular}{llcc}
\multicolumn{1}{l}{ (N) Patients } & & $\begin{array}{l}\text { Cultures in skin swabs after } \\
\mathbf{0 . 5 \%} \mathbf{C H X}+\mathbf{7 0 \%} \mathbf{A}\end{array}$ \\
& & $\mathbf{6}$ & All no growth \\
$\begin{array}{l}\text { Male- } \\
\text { Hair- }\end{array}$ & 2 & & \\
No Hair - & 4 & & All no growth \\
Female- & & $\mathbf{5}$ & \\
No Hair & 5 & &
\end{tabular}

\section{Conclusions}

In this sample of adult patients undergoing caudal epidural injections, the AAGBI guidelines for skin antisepsis proved to be effective for CNB. The caudal area has been shown to have an increased risk of colonisation when compared to lumbar site. Central neuraxial blockade does include caudal epidural injections. Larger studies is advised to assess efficacy of chlorhexidine in caudal site.

\section{References}

1- Skin antisepsis for central neuraxial blockade- AAGBI Safety Guideline 2013

2- Culture of bacteria from lumbar and caudal epidural catheters used for postoperative analgesia in children, McNeely JK et al, Reg Anesth 1997 SepOct;22(5):428-31

3- Epidural abscesses, Grewal et al, BJA: British Journal of Anaesthesia, Volume 96, Issue 3, 1 March 2006, Pages 292-302

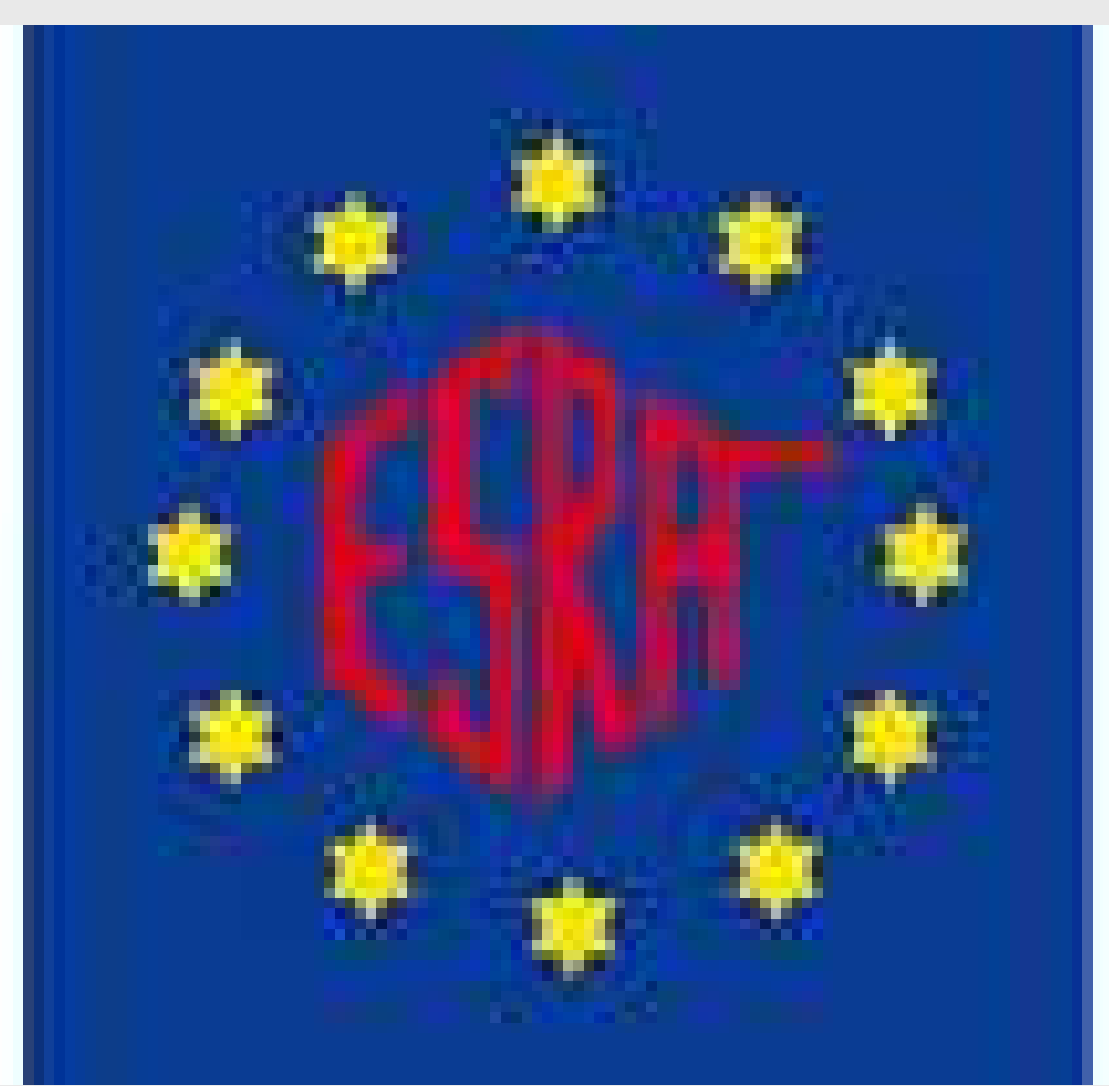

